\section{Meningitis, spondylodiscitis, pneumonia and septic shock with Streptococcus pneumoniae in a previously healthy woman with isolated IgG2-, IgG3-, IgA-deficiency and monoclonal gammopathy of undetermined significance}

\author{
Shahin Gaini, ${ }^{1-3}$ David Gudnason, ${ }^{1}$ \\ Bjarni á Steig, ${ }^{4}$ \\ Jenny Jónsdóttir Nielsen ${ }^{1}$ \\ ${ }^{1}$ Department of Medicine, Infectious \\ Diseases Division, National Hospital \\ Faroe Islands, Tórshavn, Faroe Islands; \\ ${ }^{2}$ Infectious Diseases Research Unit, \\ Odense University Hospital and \\ University of Southern Denmark, \\ Odense, Denmark; ${ }^{3}$ Department of \\ Science and Technology, University of \\ the Faroe Islands, Tórshavn, Faroe \\ Islands; ${ }^{4}$ Department of Medicine, \\ Hematology Division, National Hospital \\ Faroe Islands, Tórshavn, Faroe Islands
}

\begin{abstract}
A 66 years old Caucasian woman with pneumococcal meningitis was treated and discharged after an uncomplicated course. Five months later she was readmitted with fever and right side abdominal pain and diagnosed with pneumococcal spondylodiscitis. One year later she was treated for a severe chest X-ray confirmed left lobar pneumonia. Two years later she was diagnosed with a pneumococcal pneumonia in her left lung with septic shock. An immune deficiency screen revealed slightly reduced IgA levels, low IgG2 levels, low IgG3 levels and high IgG1 levels. No other immune defects were identified. She did not respond serologically on vaccination with 13 -valent conjugate and 23-valent polysaccharide pneumococcal vaccines. Further evaluations revealed a positive M-component in her blood and a bone marrow biopsy diagnosed her to have monoclonal gammopathy of undetermined significance. To protect her against future life threatening pneumococcal infections she was started on treatment with intravenous immunoglobulin. The case report illustrates the importance of thorough evaluation of patients with unusual infectious disease entities or unusual frequency of infections in individual patients. To optimize prophylactic measures and active treatment options in the individual patient, it is important to identify underly-
\end{abstract}

ing causes of diseases and immune deficiencies that potentially can lead to life threatening infections. This is illustrated in our case by an undiagnosed monoclonal gammopathy of undetermined significance in an apparently healthy woman with at least three life threatening documented pneumococcal infections in a two-year period and poor pneumococcal vaccine response.

\section{Introduction}

Streptococcus pneumoniae is a common invasive pathogen with pneumonia, sinuitis, otitis and meningitis as common foci of infection. It is a rare etiology of spondylodiscitis and endocarditis. Underlying diseases and immune deficiencies can increase the risk of developing invasive pneumococcal infections.

\section{Case Report}

\section{Pneumococcal infectious episode 1}

A previously healthy 66 years old Caucasian woman was admitted acutely with reported fever at home, but afebrile (36.3 degrees Celcius) at arrival to hospital and with progressive confusion. Objective findings showed photophobia, confusion and terminal neck stiffness. She was circulatory stable. Her blood chemistry showed elevated infection parameters (CRP 197 $\mathrm{mg} / \mathrm{L}$ and leukocytes $17.2 \times 10^{9} / \mathrm{L}$ ). A spinal tap showed turbid cerebrospinal fluid (CSF) with pleocytosis $\left(348 \times 10^{6} / \mathrm{L}\right)$ and $85 \%$ polynucleated leukocytes in the CSF. Levels of CSF protein was $3.9 \mathrm{~g} / \mathrm{L}$ and of CSF glucose $0.8 \mathrm{mmol} / \mathrm{L}$. The Gram stain of the CSF was negative. The patient was started in standard treatment for bacterial meningitis with intravenous Dexamethasone $(10 \mathrm{mg} \times 4)$, intravenous Benzylpenicillin $(1.8 \mathrm{~g} \times 6)$ and intravenous Ceftriaxone $(4 \mathrm{~g} \times 1)$ according to Danish guidelines. The patient had received oral Phenoxymethylpenicillin from her General Practitioner before admission, and there was no bacterial growth in the CSF. However Streptococcus pneumoniae (Serotype $15 \mathrm{C}$ ) grew in one of four blood culture bottles sampled at the time of hospital admission. The isolate was fully succeptible to Penicillin. A Chest X-ray (CXR) at the time of admission revealed a small basal pulmonary infiltration on the left side. The patient responded very well to 10 days of high dose intravenous Benzylpenicillin (1.8 $\mathrm{g} \times 6$ ) treatment and was discharged after 10 days. She was followed in the outpatient
Correspondence: Shahin Gaini, Medical Centre, Infectious Diseases Division, National Hospital Faroe Islands, J.C. Svabosgøta 4149, FO-100 Tórshavn, Faroe Islands.

Tel.: +298.304500

E-mail: shahingaini@hotmail.com

Key words: Streptococcus pneumonia, meningitis, spondylodiscitis, pneumonia, monoclonal gammopathy of undetermined significance.

Acknowledgments: the authors would thank Charlotte Sværke Jørgensen and Kurt Fuursted from Statens Serum Institute (SSI, Copenhagen, Denmark) for detailed information on the pneumococcal serology methods and results.

Contributions: SG treated and diagnosed the patient; wrote the manuscript draft; BáS diagnosed the patient and contributed to the manuscript; DG and JJN contributed to the manuscript preparation.

Conflict of interest: the authors declare no potential conflict of interest.

Received for publication: 12 July 2017.

Revision received: 13 September 2017.

Accepted for publication: 6 November 2017.

This work is licensed under a Creative Commons Attribution-NonCommercial 4.0 International License (CC BY-NC 4.0).

(C) Copyright S. Gaini, et al., 2018

Licensee PAGEPress, Italy

Infectious Disease Reports 2018; 10:7310

doi:10.4081/idr.2018.7310

clinic for a further two months. After two months she was in her normal health status with normal blood chemistry and normal levels of infection markers. She returned to her normal work as a nurse.

\section{Pneumococcal infectious episode 2}

Five months later she was admitted again acutely with reported fever at home but afebrile (36.9 degrees Celcius) at arrival to hospital. She now had pain in her right side of the abdomen stretching to the lumbar back. In the emergency room her vital parameters were stable, the abdomen was soft with no abdominal guarding and she had normal bowel sounds. CRP (133 mg/L) and leukocytes $\left(13.3 \times 10^{9} / \mathrm{L}\right)$ were elevated with neutrocytosis. Liver enzymes and alkalic phophatases were normal. She did not appear septic and was not acutely ill. The clinical picture was quite uncharacteristic and this initiated several diagnostic procedures including CXR, CT-thorax and CTabdomen with no positive findings. Her blood cultures were negative and she did 
not get any empirical antibiotic treatment while she went through diagnostic evaluations and observation admitted at the hospital. After 3 weeks of examinations and observation her blood chemistry now showed a significant elevation of the Erythrocyte Sedimentation Rate of 99. A new CXR now showed a new compression fracture of vertebra TH8 in the spine. An MRI of the spine now showed contrast enhancement at the level of TH8 and TH9 (Figure 1). Spondylodiscitis was now suspected. She was still without antibiotic treatment. A CT guided biopsy of the involved spinal segment was performed and the bone biopsy showed growth of Streptococcus pneumoniae. The isolate was fully succeptible to Penicillin. The patient was now started in treatment with intravenous Benzylpenicillin $(1.2 \mathrm{~g} \times 4)$. After four weeks of intravenous treatment she was switched over to oral Amoxicillin/Clavulanic Acid (500/125 mg $\times 3$ ) for further 6 weeks, reaching a total duration of antibiotic treatment of 10 weeks. The Streptococcus pneumoniae isolate identified in the bone biopsy from the spondylodiscitis was not kept after diagnosis, so it has not been possible to serotype this isolate and verify if the two isolates from the meningitis and the spondylodiscitis were of the same pneumococcal serotype. A transthoracic echocardiography revealed normal heart valves without signs of endocarditis. No transesophageal echocardiography was performed. The treatment was successful and her inflammatory biomarkers returned to normal levels after finishing her treatment for pneumococcal spondylodiscitis.

Eight months after the last episode with pneumococcal spondylodiscitis, the patient had no symptoms, no back pain and was again working full time as a nurse. After her pneumococcal spondylodiscitis she was vaccinated with two pneumococcal vaccines within a period of 11 months (first 13valent conjugate pneumococcal vaccine Prevenar $^{\circledR}$ and then 23-valent polysaccharide pneumococcal vaccine Pneumovax ${ }^{\circledR}$ ). Blood testing for serological vaccine response showed inadequate vaccine response to the pneumococcal vaccines indicating that they did not protect her against the pneumococcal serotypes included in both vaccines (Table 1). Control of vaccination status was performed at the Danish National Reference Laboratory for Clinical Microbiology (Statens Serum Institute, SSI, Copenhagen, Denmark) using an in-house Luminex bead-based assay. ${ }^{1}$ Antibody measurement of specific anti-pneumococcal $\mathrm{IgG}$ antibodies to serotypes $1,3,4,5,6 \mathrm{~B}, 7 \mathrm{~F}, 9 \mathrm{~V}, 14,18 \mathrm{C}$,
19A, $19 \mathrm{~F}$ and $23 \mathrm{~F}$ was performed. From the geometric mean of the 12 serotype specific antibodies, the vaccination status of the patient could be determined. After the second episode of invasive pneumococcal infection, the patient was evaluated for possible immune defects with measurements of immunoglobulins, IgG subclasses and flowcytometric analyses of her leukocyte subtypes. Low levels of IgG2, of IgG3 and of IgA levels were observed (Table 2). Very high levels of IgG1 was observed (Table 2). CT-abdomen showed a normal spleen and no signs of lymphoma. CT-thorax showed no thymoma and no signs of lymphoma. No immunological action was taken on these observed low levels of IgG2, of IgG3 and of IgA at this time.

\section{Infectious episode 3}

One year after her pneumococcal meningitis she was treated by her General Practitioner with oral antibiotics for a CXR verified large pneumonia involving the left lower lobe. CRP was measured to $125 \mathrm{mg} / \mathrm{L}$ by her General Practitioner. She was treated with Phenoxymethylpenicillin $1600 \mathrm{mg} \times 2$ for 7 days with good clinical response. No bacterial etiology was identified, but the CXR was compatible with a classic lobar pneumonia that could have been caused by Streptococcus pneumonia.

\section{Pneumococcal infectious episode 4}

Two and a half years after her pneumococcal meningitis she was admitted febrile (39.2 degrees Celcius) with septic shock and a CXR verified pneumonia. The CRP was only $18 \mathrm{mg} / \mathrm{L}$ at arrival rising to 147 $\mathrm{mg} / \mathrm{L}$ on the second day of admission. Her leukocytes at arrival were $12.4 \times 10^{9} / \mathrm{L}$ with neutrocytosis. Streptococcus pneumoniae Serotype 21 was cultured from the sputum. The isolate was fully succeptible to Penicillin. Blood cultures were negative. She was treated with Benzylpenicillin $1.2 \mathrm{~g}$

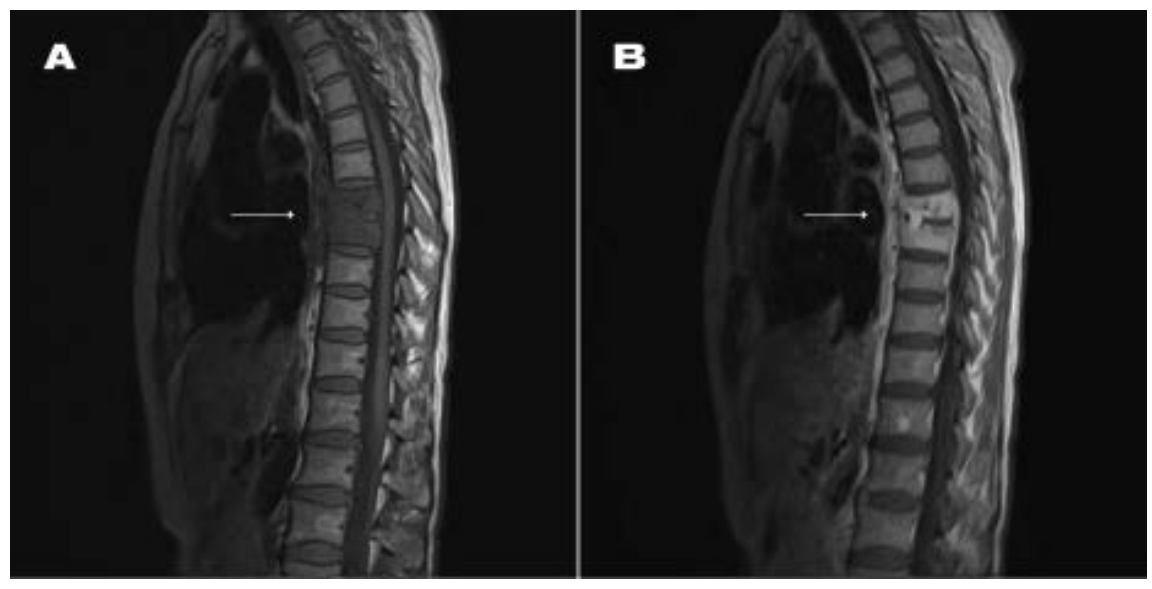

Figure 1. T1 weighted magnetic resonance imaging of the spine without (A) and with (B) intravenous contrast, shows contrast enhancement in Th8, Th9 and in the discus between (with permission from the Department of Radiology, National Hospital Faroe Islands)

Table 1. Levels of specific anti-pneumococcal IgG antibodies (mg/L).

\begin{tabular}{lccc} 
Pneumococcal serotype & September 2015 & November 2015 & May 2016 \\
Serotype 1 & 0.18 & 0.05 & 0.61 \\
Serotype 3 & 0.10 & 0.04 & 0.22 \\
\hline Serotype 4 & 0.06 & 0.02 & 0.11 \\
Serotype 5 & 0.08 & 0.04 & 0.14 \\
\hline Serotype 6B & 0.12 & 0.04 & 0.28 \\
Serotype 7F & 0.33 & 0.12 & 0.59 \\
\hline Serotype 9V & 0.43 & 0.13 & 0.28 \\
Serotype 14 & 1.02 & 0.45 & 0.84 \\
\hline Serotype 18C & 0.11 & 0.11 & 0.11 \\
Serotype 19A & 1.00 & 0.11 & 1.71 \\
\hline Serotype 19F & 0.60 & 0.07 & 2,21 \\
Serotype 23F & 1.50 & 0.09 & 1.10 \\
\hline
\end{tabular}


$\times 4$ for 5 days and after that for ten days more with Phenoxymethylpenicillin $800 \mathrm{mg}$ $\times 4$. After the last episode with life threatening septic shock with a pneumococcal pneumonia as primary focus, immune deficiency screening (immunoglobulins, IgG subclasses and flowcytometric analyses of leukocyte subtypes) was repeated (Table 2) and based on observed persistent low levels of $\operatorname{IgA}$, of $\operatorname{IgG} 2$ and of $\operatorname{IgG} 3$, intravenous immunoglobulin substitution (IVIG) therapy was started $\left(\right.$ Privigen $\left.^{\circledR}\right)$. She still had very high IgG1 levels (Table 2). She had a negative HIV test and normal CD4 counts. She has not had any serious bacterial invasive infections since starting IVIG.

In October 2016, three and a half years after her pneumococcal meningitis, a blood test showed a positive M-component. The identified M-component was an IgG-kappa chain. The identified M-component in the blood led to a bone marrow biopsy performed in December 2016. The bone marrow biopsy gave the diagnosis of monoclonal gammopathy of undetermined significance (MGUS) with 5-10\% plasma cells in the bone marrow biopsy. The patient is now followed in the hematological out-patient clinic for her MGUS and treated with IVIG to protect her against invasive infections.

\section{Discussion and Conclusions}

Streptococcus pneumoniae is a common pathogen, but pneumococcal meningitis followed by pneumococcal spondylodiscitis has been described rarely. ${ }^{2,3}$ In cases of bacteremia with Streptococcus pneumoniae, involvement of multiple organs should always be considered, especially in high risk patients (alcohol overconsumption, diabetes mellitus, immunosuppression and after splenectomi). ${ }^{4}$

Spondylodiscitis will typically have a subacute debut. Early symptoms can be unspecific and initially be back pain and fever like in our case report. ${ }^{5}$ Conventional
X-ray examinations of the spine are often normal in the early phases of spondylodiscitis. Bone scintigraphy and MRI are sensitive early in the disease process and will typically give positive findings already after few days. ${ }^{5}$ MRI is still considered as the golden standard with a high sensitivity and specificity in diagnosing spondylodiscitis. ${ }^{5}$ PET-CT is getting an increasing role in the diagnosis of spondylodiscitis and well suited for patients with contraindications for MRI. It is important to make efforts in identifying the microbiological etiology in the cases of spondylodiscitis. Positive blood cultures with a pathogen known to be associated to spondylodiscitis, combined with imaging suggesting spondylodiscitis, is usually considered as enough to have proven the etiology of the spondylodiscitis, and start a relevant antibiotic treatment strategy based on the bacterial finding. With negative blood cultures, it is important to try to perform a diagnostic bone biopsy to identify the pathogen to plan the correct antibiotic treatment. Another important reason for the bone biopsy is excluding differential diagnoses as malignant disease in the spine or tuberculosis as the cause of the spondylodiscitis (Pott's disease). ${ }^{5}$

The patient described in this case report suffered from two more severe pneumonias over the next two years after the episode of pneumococcal spondylodiscitis. This indicated that an underlying cause for infection including the possibility of immune deficiencies should be considered. An immune deficiency screening including measurements of immunoglobulins, IgG subclasses and flowcytometric analyses of the leukocyte subsets was performed. No leukocyte anomalies were observed and the CD4 count was normal, negative HIV test, negative SLE autoantibodies and no signs of lymphoma. Low IgG2 levels and low IgG3 levels were observed on several occasions, and slightly decreased $\operatorname{IgA}$ levels was also observed. The most common IgG subclass deficiency is low levels of IgG2 combined with low levels of IgA. ${ }^{6}$ The clinical significance of low $\operatorname{IgG}$ subclass deficiencies is still questionable. ${ }^{7}$ It is well known that levels of immunoglobulin and IgG subclasses can fluctuate significantly during infection. ${ }^{6}$ To make a definitive conclusion immunoglobulin levels should be measured repeatedly including in periods where the patient has no infection. It has been reported that patients with myelomatosis and MGUS have lower antibody levels against several bacteria including Streptococcus pneumonia. ${ }^{8}$ Because of the combination of several severe life threatening pneumococcal infections, in this previously healthy nurse combined with the observation of low levels of $\operatorname{IgG} 2$, of $\operatorname{IgG} 3$ and of IgA over several years, a decision was made to treat her with IVIG therapy. The combination of low levels of IgG2, of IgG3 and of IgA, and at the same time very high IgG1 levels led us to think about alternative diagnoses like myelomatosis, lymphoma, HIV and SLE. A positive M-component in her blood followed by a bone marrow biopsy revealed the presence of hematological disease in the form of MGUS. She has not suffered any serious bacterial infections after starting treatment with IVIG. Despite her age of 69 years she is still working part-time as a nurse and is healthy in all other aspects and physical active. This case report emphasis that clinicians encountering unusual infectious disease entities or unusual frequencies of infectious diseases in the same patient, should be alert regarding the possibility of underlying undiagnosed disease and immune deficiencies. Patients should be evaluated thoroughly for these risk factors regarding potential future life threatening infections in the individual patient. Identifying underlying diagnoses and immune deficiencies gives the possibility for clinical action both regarding prophylactic measures, treatment of underlying disease, treatment of underlying immune deficiencies and early rapid diagnoses of future severe infections.

Table 2. Levels of immunoglobulins and subclasses.

\begin{tabular}{|c|c|c|c|c|}
\hline Immunoglobulin, g/L (reference values) & April 2014a & September 2014 & November $2014^{c}$ & May $2015^{\mathrm{d}}$ \\
\hline IgG total (6.4-13.5) & 25.4 & 22.2 & $<0.1$ & 29.1 \\
\hline IgA total (0.7-3.12) & 0.56 & 0.41 & $<0.25$ & 0.35 \\
\hline IgM total $(0.56-3.52)$ & 1.46 & 1.28 & $<0.20$ & 1.36 \\
\hline $\operatorname{IgGl}(2.8-8)$ & 22.4 & 22.6 & 23.2 & 23.4 \\
\hline IgG2 (1.2-5.7) & 0.24 & 0.25 & 0.21 & 0.15 \\
\hline IgG3 (0.24-1.25) & 0.150 & 0.127 & 0.089 & 0.089 \\
\hline IgG4 (0.052-1.25) & 0.267 & 0.254 & 0.234 & 0.263 \\
\hline
\end{tabular}

aEight months after pneumococcal spondylodiscitis; ${ }^{\circledR} 13$ months after pneumococcal spondylodiscitis; ${ }^{\text {csampled }}$ on the $5^{\text {th }}$ day of admission with pneumococcal pneumonia with septic shock; ${ }^{d} 7$ months after pneumococcal pneumonia with septic shock. 


\section{References}

1. Lal G, Balmer P, Stanford E, et al. Development and validation of a nonaplex assay for the simultaneous quantitation of antibodies to nine Streptococcus pneumoniae serotypes. J Immunol Methods 2005;296:135-47.

2. Suzuki H, Shichi D, Tokuda Y, et al. Pneumococcal vertebral osteomyelitis at three teaching hospitals in Japan, 2003-2011: analysis of 14 cases and a review of the literature. BMC Infect Dis
2013;13:525-30.

3. Brouwer MC, de Gans J, Kuiper H, et al. Vertebral osteomyelitis complicating pneumococcal meningitis. Neurol 2008;71:612-3.

4. Turner DPJ, Weston VC, Ispahani P. Streptococcus pneumoniae spinal infection in Nottingham, United Kingdom: not a rare event. Clin Infect Dis 1999;28:873-81.

5. Lew DP, Waldvogel FA. Osteomyelitis. Lancet 2004;364:369-79.

6. Soderstrom T, Soderstrom, Hanson LA. Immunoglobulin $G$ subclasses in immunodeficiency. Ann Clin Res 1987;19:280-4.

7. Soderstrom T, Soderstrom R, Avanzini $P$, et al. Immunoglobulin $G$ subclass deficiencies. Int Arch Allergy Immunol 1987;82:476-80.

8. Karlsson J, Andréasson B, Kondori N, et al. Comparative study of immune status to infectious agents in elderly patients with multiple myeloma, Waldenstrom's macroglobulinemia, and monoclonal gammopathy of undetermined significance. Clin Vaccine Immunol 2011;18:969-77. 